\title{
Robust Adaptive Fault-Tolerant Control of Stochastic Systems with Modeling Uncertainties and Actuator Failures
}

\author{
Wenchuan Cai, ${ }^{1}$ Lingling Fan, ${ }^{1}$ and Yongduan Song ${ }^{1,2}$ \\ ${ }^{1}$ School of Electronic and Information Engineering, Beijing Jiaotong University, Beijing 100044, China \\ ${ }^{2}$ School of Automation, Chongqing University, Chongqing 400044, China \\ Correspondence should be addressed to Yongduan Song; ydsong@cqu.edu.cn
}

Received 2 January 2014; Revised 27 January 2014; Accepted 30 January 2014; Published 13 March 2014

Academic Editor: Xiaojie Su

Copyright (c) 2014 Wenchuan Cai et al. This is an open access article distributed under the Creative Commons Attribution License, which permits unrestricted use, distribution, and reproduction in any medium, provided the original work is properly cited.

\begin{abstract}
This paper deals with the problem of fault-tolerant control (FTC) of uncertain stochastic systems subject to modeling uncertainties and actuator failures. A robust adaptive fault-tolerant controller design method based on stochastic Lyapunov theory is developed to accommodate the negative impact on system performance arising from uncertain system parameters and external disturbances as well as actuation faults. There is no need for on-line fault detection and diagnosis (FDD) unit in the proposed FTC scheme, which not only simplifies the design process but also makes the implementation inexpensive. Numerical examples are provided to validate and illustrate the benefits of the proposed control method.
\end{abstract}

\section{Introduction}

Stability analysis and control design of stochastic systems have received increasing attention during the past decades. Under the framework of Itô equations together with the notion of mean-square stability, some interesting results have been obtained in terms of generalized algebraic Riccati equations, linear matrix inequality (LMI), or spectra of some operators (see, for instance, [1-4] and the references cited therein).

However, to our knowledge, very few works have dealt with the stabilization of general stochastic systems where actuator failures, parameter uncertainties, and statedependent disturbances are involved simultaneously. This motivates us to investigate the reliable control problem of stochastic systems, aiming at maintaining an acceptable performance for the closed-loop systems in the presence of actuator failures and modeling uncertainties.

Actuator failures can cause severe performance deterioration of control systems, or even system instability, leading to catastrophic accidents. Fault-tolerant control (FTC) has been viewed as one of the most promising methods to increase system safety and reliability and has thus received considerable attention from control and system engineering research community [5-17]. Most existing FTC methods can be broadly classified as active FTC and passive FTC. The active FTC requires a fault detection and diagnosis (FDD) mechanism to detect and identify the faults in real time, and a mechanism to reconfigure the controller according to the on-line fault information from the FDD [9-17]. The main idea of the passive FTC approach is to design a single controller that is robust against faults and uncertainties. In contrast to the passive approach, active methods utilize control reconfiguration to adjust controllers in real time so that the impacts of the failures can be compensated and the stability as well as the acceptable performance of the system can be maintained. Remarkable progress have been made in the area of actuator accommodation control with various effective design methods developed such as linear quadratic [18], multiple model designs [19-21], model following [2], FDD-dependent designs [22-24], and sliding mode controlbased designs $[10,25]$.

It is noted that, by blending adaptive control into FTC, the resultant control scheme turns out to be effective in reconfigurable control of systems with actuator failures [9, 26-30]. However, it is noted that few of the aforementioned works address the fault-tolerant control problem of stochastic systems. 
In this research, we will consider robust adaptive FTC for uncertain stochastic systems subject to actuator faults. The system under consideration involves parameter uncertainties and state-dependent disturbances. Moreover, there involves actuation faults that are assumed to be unpredictable during the system operation. We are interested in developing an FTC control scheme without the need for FDD. The developed FTC scheme is user friendly in the fact that no complicated computation is involved in its design and implementation.

The remaining part of the paper is organized as follows. In Section 2, the control problem is formulated. The design and analysis of the proposed control schemes are given in Section 3. Numerical simulations are conducted to demonstrate various features of the proposed control method and the results are presented in Section 4. Finally, the paper is closed with some concluding comments in Section 5.

Notation. The notations in this paper are quite standard. $R^{n}$ and $R^{n \times m}$ denote, respectively, the $n$ dimensional Euclidean space and the set of all $n \times m$ real matrices. The superscript " $T$ " denotes the transpose and the notation $X \geq Y$ (resp., $X>Y$ ) where $X$ and $Y$ are symmetric matrices, which means that $X-Y$ is positive semidefinite (resp., positive definite). $I$ is the identity matrix with compatible dimension. $|\cdot|$ is the Euclidean norm in $R^{n}$. If $A$ is a matrix, denote by $\|A\|$ its operator norm; that is, $\|A\|=\sup \{|A x|:|x|=$ $1\}=\lambda_{\max }^{1 / 2}\left(A^{T} A\right)$, where $\lambda_{\max }(\cdot)$ [resp., $\left.\lambda_{\min }(\cdot)\right]$ means the largest (resp., smallest) eigenvalues of $A$. Moreover, $(\Omega, \mathbf{F}, \mathbf{P})$ is probability space with $\boldsymbol{\Omega}$ the sample space, $\mathbf{F}$ the $\sigma$-algebra of subsets of the sample space, and $\mathbf{P}$ the probability measure. $\Xi\{\cdot\}$ stands for the mathematical expectation operator with respect to the given probability measure $\mathbf{P} . L_{2}$ and $L_{\infty}$ denote the spaces of square-integrable vector and bounded vector functions over $[0, \infty)$, respectively.

\section{Problem Statement}

Consider the stabilization problem of the following uncertain stochastic systems subject to actuator faults and external disturbances:

$$
\begin{aligned}
d x(t)= & {\left[(A+\Delta A(t)) x(t)+B\left(u_{a}(t)+f(x(t))\right)\right] d t } \\
& +(C+\Delta C(t)) x(t) d \omega(t),
\end{aligned}
$$

where $x(t) \in R^{n}$ is state, $u_{a}(t) \in R^{m}$ is actual control input, $f(x, t) \in R^{m}$ is unknown external disturbances.

Here, $\omega(t)$ is a one-dimensional Brownian motion defined on the probability space $(\boldsymbol{\Omega}, \mathbf{F}, \mathbf{P})$ with $\Xi\{\omega(t)\}=0$ and $\Xi\left\{\omega^{2}(t)\right\}=1$. A; $B$ and $C$ are known real constant matrices with appropriate dimensions. Without loss of generality, it is assumed that the pair $(A, B)$ is controllable. $\Delta A(t), \Delta C(t)$, and $C$ denote parameter uncertainties and satisfy

$$
\Delta A(t)=B F_{1}(t) \quad \Delta C(t)=B F_{2}(t) \quad C=B F_{3},
$$

where $F_{3}$ is known constant matrix, $F_{1}(t)$ and $F_{2}(t)$ are unknown time-varying matrix satisfying $\left\|F_{1}(t)\right\| \leq a_{F 1}<\infty$ and $\left(\left\|F_{2}(t)\right\|+\left\|F_{3}\right\|\right)^{2} \leq a_{F 2}<\infty$.
TABLE 1: Representations of typical actuator failures.

\begin{tabular}{lcc}
\hline Type of actuator failures & $\delta_{i}(t)$ & $\kappa(t)$ \\
\hline Healthy actuator & 1 & 0 \\
$\begin{array}{l}\text { Loss of effectiveness only } \\
\text { Loss of effectiveness and partially }\end{array}$ & $0<\delta_{i}(t) \leq 1$ & 0 \\
$\begin{array}{l}\text { out of control } \\
\text { Loss of effectiveness and partially }\end{array}$ & $0<\delta_{i}(t) \leq 1$ & Time-varying \\
jammed & Constant \\
\hline
\end{tabular}

Remark 1. It is observed that the parameter uncertainty structure as in (2) is more relaxed than the most existing methods. The parameter uncertainty structure which has been widely used in the problems of robust control and robust filtering of uncertain systems is assumed to be $\left(\Delta A(t)^{T} \Delta C(t)^{T}\right)^{T}=$ $\left(\begin{array}{ll}E_{1}^{T} & E_{2}^{T}\end{array}\right)^{T} F(t) H$, where $E_{1}, E_{2}$, and $H$ are known constant matrices and $F(t)$ is an known time-varying matrix satisfying $F^{T}(t) F(t)<I$ (see, for instance, [31-34]). Obviously, the structure herein which only needs the existence of the upper bound of $F(t)$ is easier to be satisfied.

To formulate the fault-tolerant control problem, the fault model must be established first. In system (1), the types of faults under consideration include loss of effectiveness, stuck, or combination of all. The actual control input $u_{a}(t)$ able to impact the system and the designed control input $u(t)$ designed are not the same in general. In this paper, the relationship between them will be adopted. Consider

$$
u_{a}(t)=\Delta(\cdot) u(t)+\kappa(t),
$$

where $\Delta(\cdot)=\operatorname{diag}\left\{\delta_{i}(t)\right\}$ is a diagonal matrix with $\delta_{i}(t)(i=$ $1,2, \ldots, m)$ being the unknown and time-varying scalar function called actuator efficiency factor, or "health indicator." For every fault mode, $\underline{\delta}_{i}$ and $\bar{\delta}_{i}$ represent the lower and upper bounds of $\delta_{i}$, respectively. Note that, when $\underline{\delta}_{i}=\bar{\delta}=1$, there is no fault for the $i$ th actuator $u_{i}$. When $\underline{\delta}_{i}=\bar{\delta}=0$, the $i$ th actuator $u_{i}$ is outage. When $0<\underline{\delta}_{i} \leq \overline{\bar{\delta}}<1$, the type of actuator is loss of effectiveness. $\kappa(t)$ denotes a vector function reflecting the portion of the control action produced by the actuator that is completely out of control.

The type of actuator failures considered in this work is listed in Table 1.

In order for the system to admit a feasible FTC, the following assumptions are imposed.

Assumption 2. The unparametrizable stuck-actuator fault and external disturbance are piecewise continuous bounded functions; that is, there exist unknown positive constants $a_{\kappa}$ and $a_{f}$ such that

$$
\|\kappa(t)\| \leq a_{\kappa}<\infty, \quad\|f(\cdot)\| \leq a_{f} \psi_{f}(\cdot)<\infty .
$$

Assumption 3. For the system under consideration, there exist some constants $\alpha>0$ and $\beta>0$ such that for all possible actuator faults, the following relation holds:

$$
\alpha\left\|B^{T} P x\right\|^{2} \leq \beta\left\|B^{T} P x \sqrt{\Delta(\cdot)}\right\|^{2},
$$


where

$$
\begin{gathered}
\sqrt{\Delta(\cdot)}=\operatorname{diag}\left\{\sqrt{\delta_{i}(t)}\right\}, \\
\delta_{i}(t) \in(0,1] \quad(i=1,2, \ldots, m) .
\end{gathered}
$$

Remark 4. Assumption 2 confines the vector $\kappa(t)$ and external disturbances are bounded. Assumption 3, slightly less restrictive, sets constraint on the actuation faults, which a feasible FTC is able to deal with. Clearly, such condition is well justified if all the actuators with faults are still functional (i.e., $\left.\delta_{i}(t) \neq 0\right)$, whereas the too extreme faults in that all the actuators completely fail to work (i.e., $\delta_{i}(t)=0$ ) make the assumption invalid, which, if not impossible, is significantly challenging to develop a globally stable control for the stochastic system (1); thus it is not considered in this work.

Remark 5. Since $(A, B)$ is controllable, one can choose $N_{0}$ properly such that $\bar{A}=A-B N_{0}$ is Hurwitz. Namely, for given $Q=Q^{T}>0$, there exists a symmetric and positive definite $P$ such that the following matrix inequality is established:

$$
\bar{A}^{T} P+P \bar{A}+\rho I<-Q,
$$

where $\rho=\left\|B^{T} P B\right\| a_{F 2}$. Note that we can find the proper $P$ very easily because (7) is much simpler than those complex LMIs. The method in frame of linear matrix inequality is well used in many existing works $[8,13,31,33]$.

In the end of this section, the following important lemma is given, which will be used for the development of our result.

Lemma 6 (see [35]). The trivial solution of the stochastic differential equation

$$
d x(t)=a(x, t) d t+b(x, t) d \omega,
$$

with $a(x, t)$ and $b(x, t)$ sufficiently differentiable maps, is globally asymptotically stable in probability, if there exists a positive definite, radially unbounded, twice continuously differentiable function $V(x(t), t)$ such that the infinitesimal generator is

$$
\begin{aligned}
L[V(x(t), t)]= & \frac{\partial V}{\partial t}+\left(\frac{\partial V}{\partial x}\right)^{T} a(x, t) \\
& +\frac{1}{2} b(x(t), t)^{T} \frac{\partial^{2} V}{\partial x^{2}} b(x(t), t)<0 .
\end{aligned}
$$

\section{Fault-Tolerant Control Design}

To show the idea of this work explicitly, several fault-tolerant control schemes are developed under different conditions in this section. At the beginning, a robust fault-tolerant control method is presented.

3.1. Robust Fault-Tolerant Control. In this section, a robust fault-tolerant control of the form

$$
u(t)=-N_{0} x+N(t)
$$

is proposed, where $N_{0}$ is chosen such that $A-B N_{0}$ is Hurwitz, and $N(t)$ is generated by

$$
N(t)=-\frac{a}{\lambda_{m}} \varphi(\cdot) \frac{B^{T} P x}{\left\|B^{T} P x\right\|},
$$

with $0<\lambda_{m} \leq \alpha / \beta$ being a constant, where $\lambda_{m}$ represents the lower bound of the health indicator matrix $\Delta(\cdot)$; that is, $0<\lambda_{m} \leq \lambda_{\min }(\Delta)$ and $\alpha>0, \beta>0$ are suitable constants such that

$$
\begin{gathered}
\alpha\left\|B^{T} P x\right\|^{2} \leq \beta\left\|B^{T} P x \sqrt{\Delta(\cdot)}\right\|^{2}, \\
\varphi(\cdot)=1+\left\|N_{0} x\right\|+\left\|\varphi_{f}(x)\right\|+\|x\|, \\
a=\max \left\{1, a_{N}, a_{f}, a_{F 1}\right\} .
\end{gathered}
$$

Theorem 7. Under Assumptions 2 and 3, the FTC as given in ((10a), (10b), (10c), and (10d)) exponentially stabilizes (in mean square) the stochastic system described by (1), for all admissible uncertainties as well as all actuator failures corresponding to (3).

Proof. When the system is subject to the actuator failure as described in (3), its dynamic behavior becomes

$$
\begin{aligned}
d x(t)= & {[(A+\Delta A(t)) x(t)} \\
& +B(\Delta(\cdot) u(t)+\kappa(t)+f(x(t)))] d t \\
+ & (C+\Delta C(t)) x(t) d \omega(t) .
\end{aligned}
$$

With the proposed control ((10a), (10b), (10c), (10d)), one has

$$
\begin{aligned}
d x(t)= & {[(A+\Delta A(t)) x(t)} \\
& +B(\Delta(\cdot) u(t)+\kappa(t)+f(x(t)))] d t \\
+ & (C+\Delta C(t)) x(t) d \omega(t) \\
= & {[(A+\Delta A(t)) x(t)} \\
& \left.+B\left(\Delta(\cdot)\left(-N_{0} x+N(t)\right)+\kappa(t)+f(x(t))\right)\right] d t \\
+ & (C+\Delta C(t)) x(t) d \omega(t) \\
= & \left(A-B N_{0}\right) x(t) d t \\
+ & B[\Delta(\cdot) N(t)+Z(t)] d t \\
+ & (C+\Delta C(t)) x(t) d \omega(t),
\end{aligned}
$$

where

$$
Z(\cdot)=(I-\Delta(\cdot)) N_{0} x(t)+\kappa(t)+f(\cdot)+F_{1}(t) x(t),
$$

which is bounded as

$$
\begin{aligned}
\|Z(\cdot)\| & \leq\left\|N_{0} x\right\|+\|\kappa(\cdot)\|+\|f(\cdot)\|+\left\|F_{1}(t) x(t)\right\| \\
& \leq a\left(1+\left\|N_{0} x\right\|+\|\psi(x)\|+\|x\|\right),
\end{aligned}
$$


based on Assumption 2, where $a=\max \left\{1, a_{N}, a_{f}, a_{F 1}\right\}$ and $\varphi(\cdot)=1+\left\|N_{0} x\right\|+\left\|\varphi_{f}(x)\right\|+\|x\|$. Thus, it is not difficult to get

$$
\left(B^{T} P x\right)^{T} Z \leq a \varphi(\cdot)\left\|B^{T} P x\right\| .
$$

Consider the following Lyapunov function candidate:

$$
V(x(t), t)=x^{T}(t) P x(t) .
$$

Then, by Itô's formula, the infinitesimal generator of (12) is

$$
\begin{aligned}
L[V(x(t), t)]= & x^{T}(t) P\left(A-B N_{0}\right) x(t) \\
& +\left(\left(A-B N_{0}\right) x(t)\right)^{T} P x(t) \\
& +2\left(B^{T} P x\right)^{T}\left[-\Delta(\cdot) \frac{a}{\lambda_{m}} \varphi(\cdot) \frac{B^{T} P x}{\left.\left\|B^{T} P x\right\|\right]}\right. \\
& +2\left(B^{T} P x\right)^{T} Z \\
& +x^{T}(t)(C+\Delta C(t))^{T} P(C+\Delta C(t)) x(t) .
\end{aligned}
$$

Note that the last term of (17) cannot be combined with $Z(\cdot)$; thus the adaptive updating law cannot be used to compensate its effect as usual. To establish the robust stability of the closed-loop system (12), we need to have the following development. From the fact that $\left(\left\|F_{2}(t)\right\|+\left\|F_{3}\right\|\right)^{2} \leq a_{F 2}<\infty$ and using (2), it is seen that the last term of (17) can be expressed as

$$
\begin{aligned}
& x^{T}(t)(C+\Delta C(t))^{T} P(C+\Delta C(t)) x(t) \\
& \quad=x^{T}(t)\left[B\left(F_{2}(t)+F_{3}\right)^{T} P B\left(F_{2}(t)+F_{3}\right)\right] x(t) \\
& \quad \leq x^{T}(t)\left[\left\|B^{T} P B\right\| a_{F 2}\right] x(t)
\end{aligned}
$$

from (10c), it holds that

$$
-\left(B^{T} P x\right)^{T} \Delta(\cdot)\left(B^{T} P x\right) \leq-\frac{\alpha}{\beta}\left\|B^{T} P x\right\|^{2} \leq-\lambda_{m}\left\|B^{T} P x\right\|^{2} .
$$

and by defining $\rho=\left\|B^{T} P B\right\| a_{F 2}$, the inequality (17) can be shown to satisfy

$$
\begin{aligned}
L[V(x(t), t)] \leq & x^{T}(t)\left(\bar{A}^{T} P+P \bar{A}\right) x(t) \\
& -2 \frac{a}{\lambda_{m}} \frac{\varphi(\cdot)}{\left\|B^{T} P x\right\|}\left(B^{T} P x\right)^{T} \Delta(\cdot)\left(B^{T} P x\right) \\
& +a \varphi(\cdot)\left\|B^{T} P x\right\|+x^{T}(t)\left\|B^{T} P B\right\|\left\|F_{2}\right\|^{2} x(t) \\
\leq & x^{T}(t)\left(\bar{A}^{T} P+P \bar{A}+\rho I\right) x(t) \\
\leq & -\frac{1}{2} x^{T} Q x<0 \text { for } x(t) \neq 0,
\end{aligned}
$$

where matrixes $P$ and $Q$ are chosen properly to satisfy $\bar{A}^{T} P+P \bar{A}+\rho I \leq-Q$. Therefore, it is confirmed from Lemma 6 that the closed-loop system (11) is asymptotically mean square stable in probability despite faulty actuators with the proposed FTC.

Remark 8. Note that if proper constants $\alpha$ and $\beta$ can be obtained in advance, the proposed control ((10a), (10b), (10c), and (10d)) achieved exponential stability in mean square for the stochastic system under Assumptions 2 and 3 . However, it is a little difficult to select such $\alpha$ and $\beta$ to ensure $\lambda_{m} \leq \alpha / \beta$, since $\lambda_{m}$ the lower bound of the eigenvalue of the health indicator matrix is not available in general. In view of this, a more feasible method is developed in the next subsection.

3.2. Robust Adaptive Fault-Tolerant Control. In order to develop a control scheme that is not only robust but also adaptive yet fault-tolerant, we modify the previous one to get

$$
u(t)=-N_{0} x+\widehat{N}(t)
$$

where $N_{0}>0$ is chosen such that $A-B N_{0}$ is Hurwitz and $\widehat{N}(t)$ is on-line updated by

$$
\widehat{N}(t)=\frac{\widehat{a}(t) \varphi(x) B^{T} P x}{\left\|B^{T} P x\right\|},
$$

with

$$
\begin{gathered}
\varphi(x)=1+\left\|N_{0} x\right\|+\left\|\varphi_{f}(x)\right\|+\|x\|, \\
\dot{\hat{a}}(t)=-\gamma \varphi(x)\left\|B^{T} P x\right\|, \quad \gamma>0 .
\end{gathered}
$$

Theorem 9. Consider the uncertain stochastic system (11) under Assumptions 2 and 3. If the robust adaptive fault-tolerant controller ((21a), (21b), (21c), and (21d)) is implemented, the closed-loop system is ensured to be asymptotically stable.

Proof. Substituting the proposed control ((21a), (21b), (21c), and (21d)) into the stochastic system (11), we obtain the closed-loop system dynamics as follows:

$$
\begin{aligned}
d x(t)= & {[(A+\Delta A(t)) x(t)} \\
& +B(\Delta(\cdot) u(t)+\kappa(t)+f(x(t)))] d t \\
+ & (C+\Delta C(t)) x(t) d \omega(t) \\
= & {[(A+\Delta A(t)) x(t)} \\
& \left.+B\left(\Delta(\cdot)\left(-N_{0} x+\widehat{N}(t)\right)+\kappa(t)+f(x(t))\right)\right] d t \\
& +(C+\Delta C(t)) x(t) d \omega(t) \\
= & \left(A-B N_{0}\right) x(t) d t \\
& +B[\Delta(\cdot) \widehat{N}(t)+\mathrm{Z}(t)] d t \\
& +(C+\Delta C(t)) x(t) d \omega(t) .
\end{aligned}
$$


Consider the following Lyapunov function candidate:

$$
V(x(t), t)=x^{T} P x+\frac{1}{\lambda_{m} \gamma}\left(a-\widehat{a} \lambda_{m}\right)^{2},
$$

where $\gamma>0$ is a constant related to adaptation rate chosen by the designer and $\lambda_{m}>0$ is constant defined as before. Upon using the control scheme with the adaptive algorithm, it is not difficult to show that

$$
\begin{aligned}
L[V(x(t), t)]= & x^{T}(t) P\left(A-B N_{0}\right) x(t) \\
& +\left(\left(A-B N_{0}\right) x(t)\right)^{T} P x(t) \\
& +2\left(a-\widehat{a} \lambda_{m}\right)\left(-\dot{\hat{a}} \gamma^{-1}\right) \\
& +x^{T}(t)(C+\Delta C(t))^{T} P(C+\Delta C(t)) x(t) \\
= & {[\bar{A} x+B(\Delta(\cdot) \widehat{N}(t) x+Z(\cdot))]^{T} P x } \\
& +x^{T} P[\bar{A} x+B(\Delta(\cdot) \widehat{N}(t) x+Z(\cdot))] \\
& +2\left(a-\widehat{a} \lambda_{m}\right)\left(-\dot{\hat{a}} \gamma^{-1}\right) \\
& +x^{T}(t)(C+\Delta C(t))^{T} P(C+\Delta C(t)) x(t) \\
= & x^{T}(\bar{A} P+P \bar{A}) x \\
& +2 x^{T} P B(\Delta(\cdot) \widehat{N}(t) x+Z(\cdot)) \\
& +2\left(a-\widehat{a} \lambda_{m}\right)\left(-\dot{\hat{a}} \gamma^{-1}\right) \\
& \\
& \\
& \\
& \\
& \\
&
\end{aligned}
$$

Then

$$
\begin{aligned}
L[V(x(t), t)] \\
=x^{T}\left(\bar{A}^{T} P+P \bar{A}\right) x \\
\quad+2 x^{T} P B\left\{\Delta(\cdot)\left[-\frac{\widehat{a} \varphi(x)\left(B^{T} P x\right)}{\left\|B^{T} P x\right\|}\right]+Z(\cdot)\right\} \\
\quad+2\left(a-\lambda_{m} \widehat{a}\right)\left(-\gamma^{-1} \dot{\hat{a}}\right) \\
\quad+x^{T}(t)(C+\Delta C(t))^{T} P(C+\Delta C(t)) x(t) .
\end{aligned}
$$

In light of the definition of $\lambda_{m}$, it is true that $\left(B^{T} P x\right)^{T}$ $\Delta(\cdot)\left(B^{T} P x\right) \geq \lambda_{m}\left\|B^{T} P x\right\|^{2}$; thus the second term in (25) can be rewritten as

$$
\begin{aligned}
2 x^{T} P B & \left\{\Delta(\cdot)\left[-\frac{\widehat{a} \varphi(x)\left(B^{T} P\right)}{\left\|B^{T} P x\right\|}\right] x+Z(\cdot)\right\} \\
= & -2 \frac{\widehat{a} \varphi(x)}{\left\|B^{T} P x\right\|}\left(B^{T} P x\right)^{T} \Delta(\cdot)\left(B^{T} P x\right) \\
& +2\left(B^{T} P x\right)^{T} Z(\cdot) \\
\leq & 2\left(a-\lambda_{m} \widehat{a}\right) \varphi(x)\left\|B^{T} P x\right\| .
\end{aligned}
$$

The fact $x^{T}(t)(C+\Delta C(t))^{T} P(C+\Delta C(t)) x(t) \leq$ $x^{T}(t)\left[\left\|B^{T} P B\right\| a_{F 2}\right] x(t) \leq x^{T}(t)(\rho I) x(t)$ leads $(25)$ to

$$
\begin{aligned}
L[V(x(t), t)] \leq & x^{T}(t)\left(\bar{A}^{T} P+P \bar{A}\right) x(t) \\
& +2\left(a-\lambda_{m} \widehat{a}\right) \varphi(x)\left\|B^{T} P x\right\| \\
& +2\left(a-\lambda_{m} \widehat{a}\right)\left(-\gamma^{-1} \dot{\hat{a}}\right) \\
& +x^{T}(t)\left(\left\|B^{T} P B\right\|\left\|F_{2}\right\|^{2}\right) x(t) \\
\leq & x^{T}(t)\left(\bar{A}^{T} P+P \bar{A}+\rho I\right) x(t) .
\end{aligned}
$$

Using the updating law (21d) and choosing the proper matrixes $P$ and $Q$ to ensure that the matric inequality is established, one obtains from (27) that

$$
L[V(x(t), t)] \leq-\frac{1}{2} x^{T} Q x<0 \quad \text { for } x(t) \neq 0 .
$$

Consequently, according to Lemma 6, it can be obtained that the closed-loop system (11) is globally asymptotically stable in probability in presence of actuator failures.

Remark 10. Note that in designing and implementing the first robust fault-tolerant control method we need to predetermine the parameters $a$ and $\lambda_{m}$. This might present analytical and technical difficulty in practice. The second robust adaptive FTC scheme, which does not need the analytic computation of the parameters $a$ and $\lambda_{m}$, circumvents this shortcoming. Although the existence of $\lambda_{m}>0$ is used in stability analysis, none of them are used in the control algorithm.

Remark 11. It is seen that the proposed control is independent of explicit information on faults and disturbances. As with most variable structure control methods, when the states get closer to zero, the control scheme might experience chattering, which can be easily avoided by replacing $z /\|z\|$ with $z /(\|z\|+\varsigma)$, where $\varsigma$ is a small number, as commonly 
adopted in the literature. Also to prevent the estimate $\widehat{a}$ from drifting, (21d) can be modified as

$$
\dot{\hat{a}}(t)=-\sigma \widehat{a}+\gamma \frac{\varphi(x)^{2}\left\|B^{T} P x\right\|^{2}}{\varphi(x)\left\|B^{T} P x\right\|+\varsigma}, \quad \gamma>0, \sigma>0 .
$$

In this case, we have the following ultimately uniformly bounded (UUB) stabilization result.

Theorem 12. Consider the uncertain stochastic system (11). Let the Assumptions 2 and 3 hold. If the following robust adaptive control is applied:

$$
u(t)=-N_{0} x+\widehat{N}(t)
$$

where $N_{0}>0$ is chosen such that $A-B N_{0}$ is Hurwitz, and $\widehat{N}(t)$ is generated by

$$
\widehat{N}(t)=\frac{\widehat{a}(t) \varphi(x)^{2} B^{T} P x}{\left\|B^{T} P x\right\| \varphi(x)+\varsigma}
$$

and $\hat{a}$ is updated by (29a), then the closed-loop system (11) is ensured to UUB stable.

Proof. The result can be established by using the method similar to that as in [15].

Remark 13. Since the robust FTC with the fixed gain may bring more conservatives, a new robust adaptive FTC is further addressed in the next subsection. By means of the online estimation of effectiveness values of faulty actuators, the robust adaptive FTC gain is adaptively updated to compensate the effects of actuator faults.

3.3. Improved Robust Adaptive Fault-Tolerant Control. Consider that the elements of the actuator efficiency factor $\Delta(\cdot)$ are constants. A robust and adaptive control scheme integrated with on-line fault estimation is designed as

$$
u(t)=-\widehat{\Delta}(t)^{-1} N_{0} x+\widehat{N}(t)
$$

where $\widehat{\Delta}(t)=\operatorname{diag}\left\{\widehat{\delta}_{1}(t), \widehat{\delta}_{2}(t), \ldots, \widehat{\delta}_{m}(t)\right\}, \widehat{\delta}_{i}(t)$ is the estimated values of effectiveness for $i$ th actuator, and the updating law for $\widehat{\delta}_{i}(t)(i=1,2, \ldots, m)$ is given as

$$
\dot{\hat{\delta}}_{i}(t)=\underset{\left[\underline{\delta}_{i}, \bar{\delta}_{i}\right]}{\operatorname{Pr}} \begin{cases}0, & \text { if } \widehat{\delta}_{i}=\underline{\delta}_{i}, U_{i} \leq 0, \text { or } \widehat{\delta}_{i}=\bar{\delta}_{i}, U_{i} \geq 0 \\ U_{i}, & \text { otherwise, }\end{cases}
$$

where $U_{i}=\eta_{i} x(t)^{T}(P B)_{i} \widehat{\Delta}(\cdot)^{-1} N_{0}^{i} x(t), \eta_{i}>0$ is the adaptive law gain to be chosen according to practical applications. Here, $M^{i}$ and $M_{i}$ denote the $i$ th row and $i$ th column of a matrix $M$, respectively.

$N_{0}>0$ is chosen such that $A-B N_{0}$ is Hurwitz, and $\widehat{N}(t)$ is on-line updated by

$$
\widehat{N}(t)=\frac{\widehat{a}(t) \psi(x) B^{T} P x}{\left\|B^{T} P x\right\|},
$$

with

$$
\begin{gathered}
\psi(x)=1+\left\|\varphi_{f}(x)\right\|+\|x\|, \\
\dot{\hat{a}}(t)=-\gamma \psi(x)\left\|B^{T} P x\right\|, \quad \gamma>0 .
\end{gathered}
$$

Remark 14. It is noted from (30b) that $\operatorname{Pr}\{\cdot\}$ is a projection operator [28], which projects the estimate $\widehat{\delta}_{i}$ into the interval $\left[\underline{\delta}_{i}, \bar{\delta}_{i}\right]$ so as to satisfy the assumption on the bound of effectiveness values in (3). Because this updating law can ensure the estimated values $\widehat{\delta}_{i}(t)$ are not zero, the control signal $u(t)$ will take effect on the plant.

Theorem 15. For the uncertain stochastic system (11), the robust adaptive fault-tolerant controller given as ((30a), (30b), $(30 \mathrm{c}),(30 \mathrm{~d})$, and $(30 \mathrm{e}))$ can ensure that the state will asymptotically tend to zero.

Proof. Substituting ((30a), (30b), (30c), (30d), and (30e)) into the stochastic system (11), we obtain the closed-loop system equation as follows:

$$
\begin{aligned}
d x(t)= & {[(A+\Delta A(t)) x(t)} \\
& +B(\Delta(\cdot) u(t)+\kappa(t)+f(x(t)))] d t \\
+ & (C+\Delta C(t)) x(t) d \omega(t) \\
= & {[(A+\Delta A(t)) x(t)} \\
& +B\left(\Delta(\cdot)\left(-\widehat{\Delta}(\cdot)^{-1} N_{0} x+\widehat{N}(t)\right)\right. \\
& \quad+\kappa(t)+f(x(t)))] d t \\
& +(C+\Delta C(t)) x(t) d \omega(t) \\
= & \left(A-B N_{0}\right) x(t) d t \\
& +B\left[\left(I-\Delta(\cdot) \widehat{\Delta}(\cdot)^{-1}\right) N_{0} x+\Delta(\cdot) \widehat{N}(t)+Z(\cdot)\right] d t \\
& +(C+\Delta C(t)) x(t) d \omega(t),
\end{aligned}
$$

where $Z(\cdot)=\kappa(t)+f(\cdot)+F_{1}(t) x(t)$, which is bounded by

$$
\begin{aligned}
\|Z(\cdot)\| & \leq\|\kappa(\cdot)\|+\|f(\cdot)\|+\left\|F_{1}(t) x(t)\right\| \\
& \leq a(1+\|\varphi(x)\|+\|x\|)
\end{aligned}
$$

under Assumption 2.

Consider the following Lyapunov function candidate

$$
V(x(t), t)=x^{T} P x+\frac{1}{\lambda_{m} \gamma}\left(a-\widehat{a} \lambda_{m}\right)^{2}+\sum_{i=1}^{m} \eta_{i}^{-1} \widetilde{\delta}_{i}^{2}(t)
$$

where $\gamma>0$ and $\eta>0$ are constants related to adaptation rate chosen by the designer and $\lambda_{m}>0$ is constant defined as before. Upon using the control scheme with the adaptive 
algorithm, it is not difficult to show that the infinitesimal operator

$$
\begin{aligned}
L[V(x(t), t)]= & x^{T}(t) P\left(A-B N_{0}\right) x(t) \\
& +\left(\left(A-B N_{0}\right) x(t)\right)^{T} P x(t) \\
& +2\left(a-\widehat{a} \lambda_{m}\right)\left(-\dot{\hat{a}} \gamma^{-1}\right) \\
& +2 \sum_{i=1}^{m} \eta_{i}^{-1} \widetilde{\delta}_{i}(t) \dot{\widehat{\delta}}_{i}(t) \\
= & {\left[\bar{A} x+B\left(-\widetilde{\delta} \widehat{\delta}^{-1} N_{0} x\right.\right.} \\
& \left.+\Delta(\cdot) \widehat{N}(t) x+Z(\cdot))^{T}\right] P x \\
& +x^{T} P\left[\bar{A} x+B\left(-\widetilde{\delta} \widehat{\delta}^{-1} N_{0} x\right.\right. \\
& +\Delta(\cdot) \widehat{N}(t) x+Z(\cdot))] \\
& +2\left(a-\widehat{a} \lambda_{m}\right)\left(-\dot{\hat{a}} \gamma^{-1}\right) \\
& +2 \sum_{i=1}^{m} \eta_{i}^{-1} \widetilde{\delta}_{i}(t) \dot{\hat{\delta}_{i}}(t) \\
= & x^{T}\left(\bar{A}^{T} P+P \bar{A}\right) x+2 x^{T} P B \widetilde{\delta} \widehat{\delta}^{-1} N_{0} x \\
& +2 x^{T} P B(\Delta(\cdot) \widehat{N}(t) x+Z(\cdot)) \\
& +2\left(a-\widehat{a} \lambda_{m}\right)\left(-\dot{\hat{a}} \gamma^{-1}\right) \\
& +2 \sum_{i=1}^{m} \eta_{i}^{-1} \widetilde{\delta}_{i}(t) \dot{\dot{\delta}_{i}}(t) .
\end{aligned}
$$

Considering that $P B \widetilde{\delta} \widehat{\delta}^{-1}=\sum_{i=1}^{m} \widetilde{\delta}(P B)^{i} \widehat{\delta}^{-1}$ and the adaptive law (30b), we have

$$
-2 x^{T} P B \widetilde{\delta} \widehat{\delta}^{-1} N_{0} x+2 \sum_{i=1}^{m} \eta_{i}^{-1} \widetilde{\delta}_{i}(t) \dot{\hat{\delta}}_{i}(t) \leq 0
$$

Then, $L[V(x(t), t)]$ becomes

$$
\begin{aligned}
L[V & (x(t), t)] \\
= & x^{T}\left(\bar{A}^{T} P+P \bar{A}\right) x \\
& +2 x^{T} P B\left\{\Delta(\cdot)\left[-\frac{\widehat{a} \psi(x)\left(B^{T} P x\right)}{\left\|B^{T} P x\right\|}\right]+Z(\cdot)\right\} \\
& +2\left(a-\lambda_{m} \widehat{a}\right)\left(-\gamma^{-1} \dot{\hat{a}}\right) \\
& +x^{T}(t)(C+\Delta C(t))^{T} P(C+\Delta C(t)) x(t),
\end{aligned}
$$

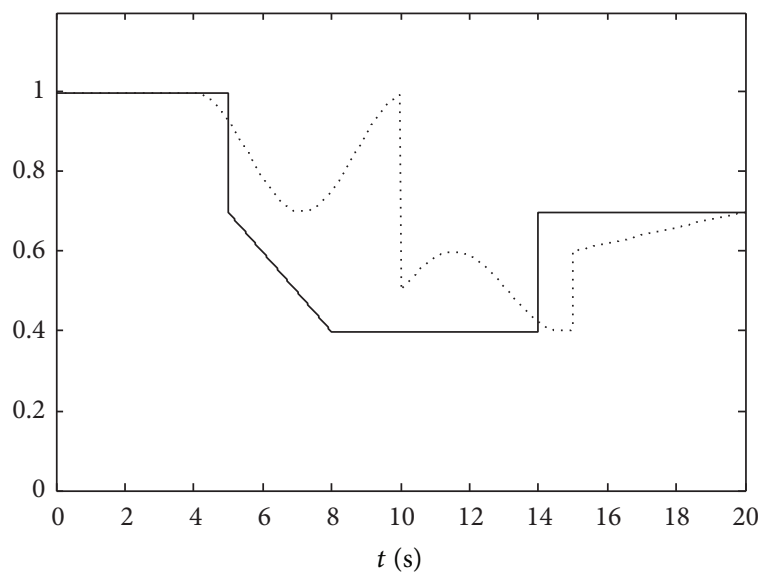

FIgure 1: Profile of the time-varying actuator efficiency variable $\left(\delta_{1}\right.$ (solid), $\delta_{2}($ dot $)$ ).

in which the second term in (36) can be rewritten as

$$
\begin{aligned}
2 x^{T} P B & \left\{\Delta(\cdot)\left[-\frac{\widehat{a} \psi(x)\left(B^{T} P\right)}{\left\|B^{T} P x\right\|}\right] x+Z(\cdot)\right\} \\
= & -2 \frac{\widehat{a} \psi(x)}{\left\|B^{T} P x\right\|}\left(B^{T} P x\right)^{T} \Delta(\cdot)\left(B^{T} P x\right) \\
& +2\left(B^{T} P x\right)^{T} Z(\cdot) \\
\leq & 2\left(a-\lambda_{m} \widehat{a}\right) \psi(x)\left\|B^{T} P x\right\| ;
\end{aligned}
$$

by using (19) it is true that $\left(B^{T} P x\right)^{T} \Delta(\cdot)\left(B^{T} P x\right) \geq \lambda_{m}\left\|B^{T} P x\right\|^{2}$. Thus by using the fact that $x^{T}(t)(C+\Delta C(t))^{T} P(C+$ $\Delta C(t)) x(t) \leq x^{T}(t)(\rho I) x(t)$ and the updating law (30e), the function $L[V(x(t), t)]$ eventually is bounded as

$$
\begin{aligned}
L[V(x(t), t)] \leq & x^{T}\left(\bar{A}^{T} P+P \bar{A}+\rho I\right) x \\
& +2\left(a-\lambda_{m} \widehat{a}\right) \psi(x)\left\|B^{T} P x\right\| \\
& +2\left(a-\lambda_{m} \widehat{a}\right)\left(-\gamma^{-1} \dot{\hat{a}}\right) \\
\leq & -\frac{1}{2} x^{T} Q x<0 \quad \text { for } x(t) \neq 0,
\end{aligned}
$$

as long as proper matrixes $P$ and $Q$ are select to ensure (7). Therefore, it can be obtained from Lemma 6 that the state of the stochastic system is asymptotically stable in probability and the estimation parameters $\left(a-\lambda_{m} \widehat{a}\right)$ and $\widetilde{\delta}_{i}$ are bounded.

\section{Numerical Simulation}

Two examples are used to demonstrate the features of the proposed control scheme. 


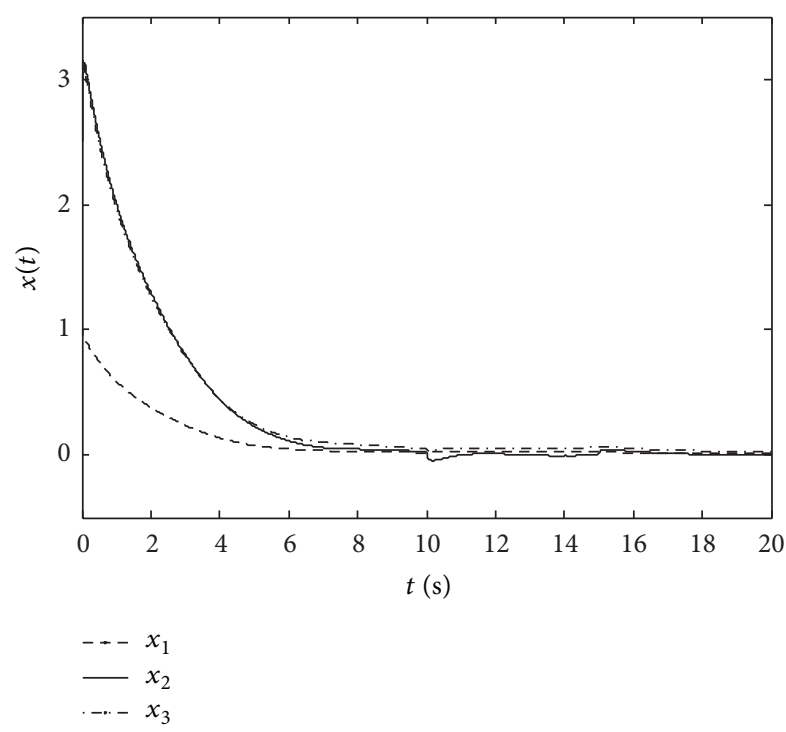

FIGURE 2: The curve of $x(t)$ with the proposed control scheme ((29a), (29b), and (29c)).

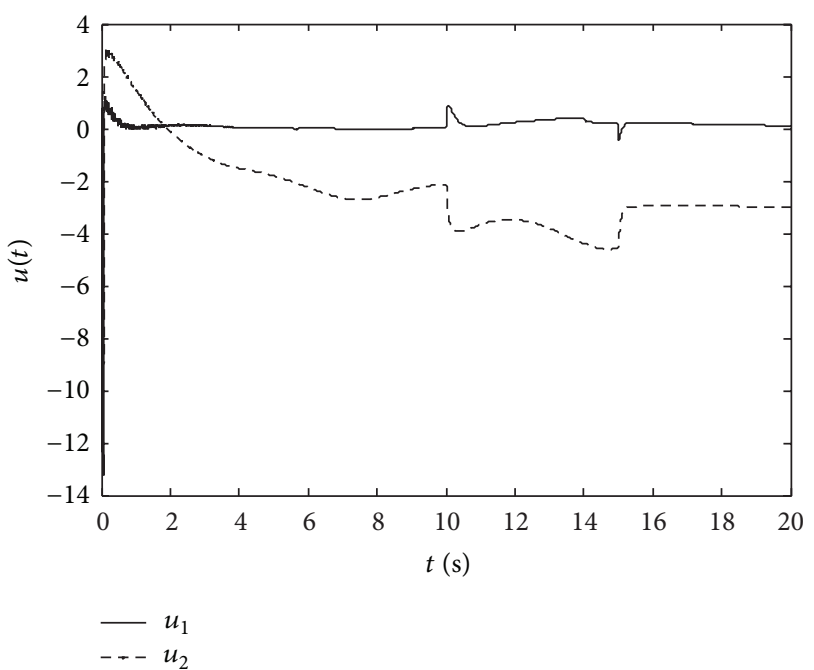

FIGURE 3: The curve of $u(t)$ with the proposed control scheme ((29a), $(29 b)$, and (29c)).

Example 1. Consider the uncertain stochastic system (11) with

$$
\begin{gathered}
A=\left[\begin{array}{ccc}
0.2 & -0.2 & 0 \\
-0.2 & -0.6 & 0.3 \\
0.2 & -0.4 & -0.2
\end{array}\right], \quad B=\left[\begin{array}{cc}
-0.2 & 0.2 \\
1 & -1.7 \\
0.6 & -0.7
\end{array}\right], \\
C=\left[\begin{array}{ccc}
-0.04 & 0.2 & 0.07 \\
-0.03 & 0.1 & 0.04 \\
0.04 & -0.2 & -0.07
\end{array}\right], \\
\Delta A=\left[\begin{array}{ccc}
0.02 \sin (t) & 0.04 \cos ^{2}(t) & 0.04 \cos (2 t) \\
0.02 & 0.04 \sin (2 t) \cos (t) & 0.04 \\
0.03 \cos (t) & 0.06 & 0.06 \sin (t)
\end{array}\right],
\end{gathered}
$$

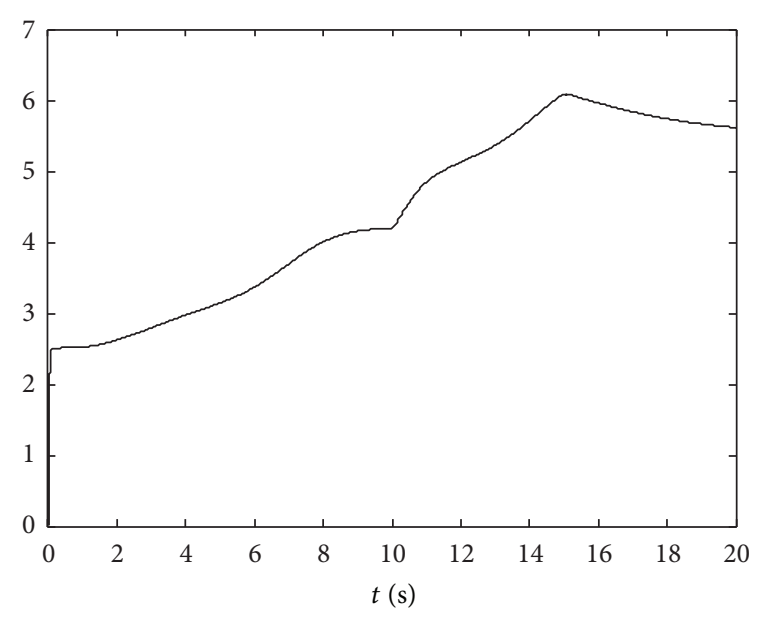

FIGURE 4: Updating of $\widehat{a}(t)$ with the proposed control scheme ((29a), $(29 b)$, and (29c)).

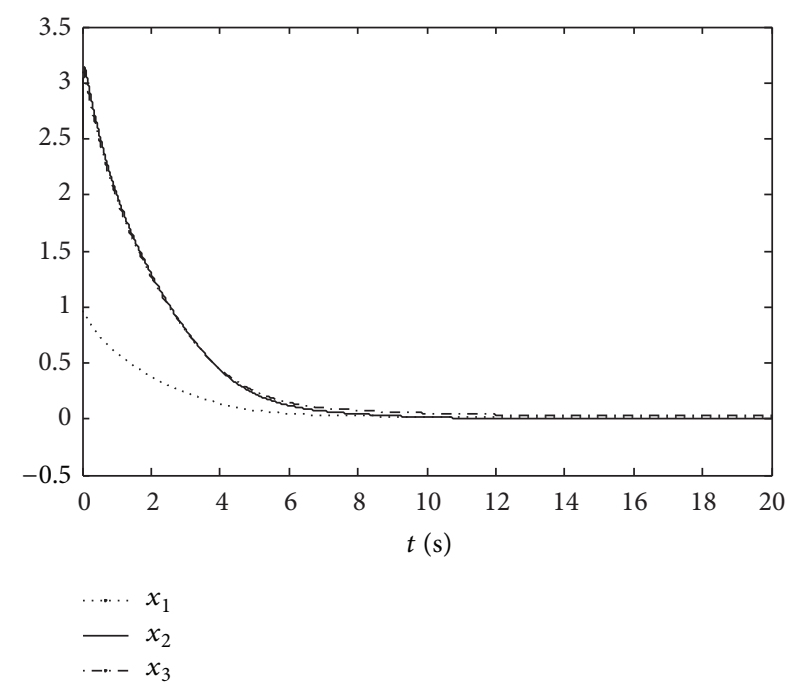

FIGURE 5: System responses under the control of the proposed FTC ((30a), (30b), (30c), (30d), and (30e)).

$$
\begin{gathered}
\Delta C=\left[\begin{array}{ccc}
-0.007 \cos (t) & 0.014 & 0.014 \sin (2 t) \\
0.004 \cos ^{2}(t) & 0.008 \sin ^{2}(t) & 0.008 \\
-0.007 & -0.014 \sin (t) \cos (2 t) & -0.014 \sin (t) \cos (3 t)
\end{array}\right], \\
f(x(t))=\left(\begin{array}{c}
\sin \left(x_{1}(t)\right) \sin \left(x_{2}(t)\right) \\
2 x_{1}(t) \cos \left(x_{2}(t)\right)
\end{array}\right) .
\end{gathered}
$$

It is seen that the uncertainties $\Delta A$ and $\Delta C$ are complex to be described by the form of $\left(\Delta A(t)^{T} \Delta C(t)^{T}\right)^{T}=$ $\left(\begin{array}{ll}E_{1}^{T} & E_{2}^{T}\end{array}\right)^{T} F(t) H$. But the form of (2) is easy to satisfy. The external disturbance $f(\cdot)$ is state-dependent and unknown. For the simulation, the initial conditions are $x(0)=[1,2.5,3]$ and $\widehat{a}(0)=0$.

The actuator efficiency variables for each of the two control channels simulated are as illustrated in Figure 1, where two of the actuators suffer from the failure as shown in the figure. 


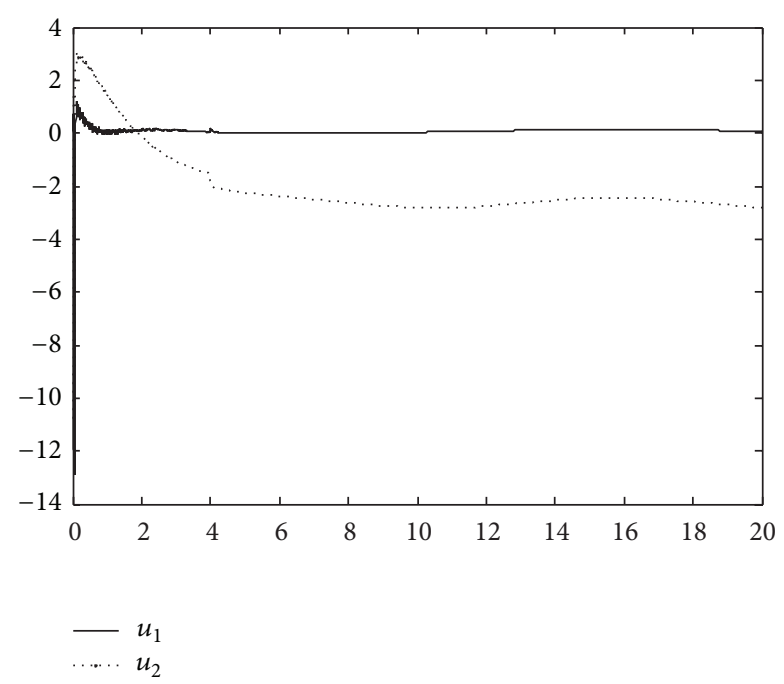

Figure 6: The curve of $u(t)$ with the proposed control scheme ((30a), (30b), (30c), (30d), and (30e)).

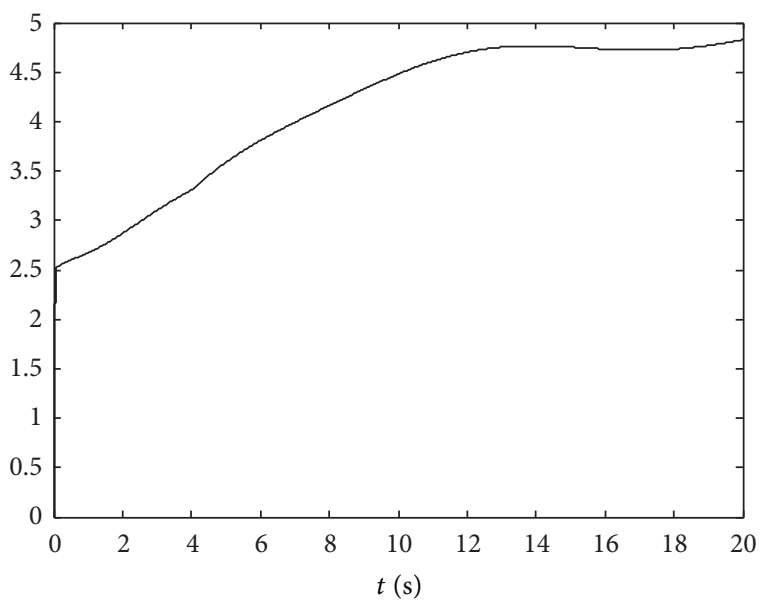

FIGURE 7: Updating of $\widehat{a}(t)$ with the proposed control scheme ((30a), (30b), (30c), (30d), and (30e)).



FIGURE 8: The curve of the estimate $\widehat{\Delta}(t)$ with $\eta=2\left(\widehat{\delta}_{1}\right.$ (solid), $\widehat{\delta}_{2}$ (dot)).
The scenario simulated is that the system operates normally at the beginning, and the disturbances always exist during the system operation. After 4 seconds of the operation some faults in actuators occur: the first and the second actuators encounter severe failures in the fact that both channels lose their effectiveness by over $50 \%$ at some time and the faults are fast time-varying for some period.

The objective in this work is to design a reliable robust adaptive fault-tolerant controller such that the closed-loop system is asymptotically stable in probability despite the presence of actuator faults. In applying the control scheme ((29a), (29b), and (29c)), one can easily determine all the control parameters:

$$
\begin{gathered}
N_{0}=\left[\begin{array}{ccc}
-1.0912 & 0.3210 & 0.0695 \\
8.0245 & -3.8070 & 0.0239
\end{array}\right], \\
\gamma=5, \quad \sigma=0.08, \quad \varepsilon=0.001, \\
\varphi(x)=1+\left\|N_{0} x\right\|+\left\|\varphi_{f}(x)\right\|+\|x\| .
\end{gathered}
$$

The simulation results in terms of stabilization of the three states are presented in Figure 2. It can be seen that the states $x_{1}, x_{2}$, and $x_{3}$ can converge to a small neighborhood around zero. Figure 3 shows the control signals of the two inputs. The estimated parameter $\widehat{a}(t)$ is shown in Figure 4. The results confirm the theoretical prediction.

Example 2. The second simulation is made for robust adaptive fault-tolerant controller ((30a), (30b), (30c), (30d), and $(30 \mathrm{e}))$. It is assumed that at $t=4 \mathrm{~s}$, the first actuator $u_{1}$ is still normal and the second actuator $u_{2}$ is faulty with $\delta_{2}=0.5$. The simulations are shown in Figures 5, 6, 7, and 8. Also it should be pointed out from [36] that the estimated value $\widehat{\delta}_{i}(t)(i=$ $1,2)$ can converge but may not converge to its true value $\delta_{i}(t)$. And in our controller design procedure, only the estimated value $\widehat{\delta}_{i}(t)$ is needed to construct adaptive controller and whether $\widehat{\delta}_{i}(t)$ can converge to its true values or not is not necessary.

From Figure 5, the FTC scheme ((30a), (30b), (30c), (30d), and (30e)) makes the curves relatively smooth via the adaptive estimate $\widehat{\delta}_{i}(t)$ of efficiency value. The simulation results confirm that the robust adaptive FTC can achieve a good performance on dealing with the reliable control problem of stochastic systems in presence of actuator failures, parameter uncertainty, and state-dependent disturbance.

\section{Conclusion}

In this paper, the problem of robust adaptive FTC for stochastic systems with faulty actuators has been considered. By blending adaptive control into robust FTC, the proposed control method is able to accommodate actuation faults and modeling uncertainties concurrently. Both theoretical analysis and numerical simulations validate the benefits and effectiveness of the proposed approach. 


\section{Conflict of Interests}

The authors declare that there is no conflict of interests regarding the publication of this paper.

\section{Acknowledgments}

The paper is supported by the Major State Basic Research Development Program 973 (no. 2012CB215202), the National Natural Science Foundation of China (no. 61134001 and 61203124), and Fundamental Research Funds for the Central Universities (2013YJS021).

\section{References}

[1] D. Hinrichsen and A. J. Pritchard, "Stochastic $H_{\infty}$ ", SIAM Journal on Control and Optimization, vol. 36, no. 5, pp. 15041538, 1998.

[2] J. Zhang, M. Ren, Y. Tian, G. Hou, and F. Fang, "Constrained stochastic distribution control for nonlinear stochastic systems with non-Gaussian noises," International Journal of Innovative Computing, Information and Control, vol. 9, no. 4, pp. 1759-1767, 2013.

[3] I. R. Petersen, V. A. Ugrinovskii, and A. V. Savkin, Robust control design using $H_{\infty}$-methods, Springer, London, UK, 2000.

[4] X. Su, L. Wu, P. Shi, and Y. D. Song, " $H_{\infty}$ model reduction of Takagi-Sugeno fuzzy stochastic systems," IEEE Transactions on Systems, Man, and Cybernetics B, vol. 42, no. 6, pp. 1574-1585, 2012.

[5] A. S. Willsky, "A survey of design methods for failure detection in dynamic systems," Automatica, vol. 12, no. 6, pp. 601-611, 1976.

[6] L.-L. Fan and Y.-D. Song, "Neuro-adaptive model-reference fault-tolerant control with application to wind turbines," IET Control Theory and Applications, vol. 6, no. 4, pp. 475-486, 2012.

[7] R. J. Veillette, J. B. Medanic, and W. R. Perkins, "Design of reliable control systems," IEEE Transactions on Automatic Control, vol. 37, no. 3, pp. 290-304, 1992.

[8] Z. Wang, B. Huang, and K. J. Burnham, "Stochastic reliable control of a class of uncertain time-delay systems with unknown nonlinearities," IEEE Transactions on Circuits and Systems, vol. 48, no. 5, pp. 646-650, 2001.

[9] G. Tao, S. M. Joshi, and X. L. Ma, "Adaptive state feedback and tracking control of systems with actuator failures," IEEE Transactions on Automatic Control, vol. 46, no. 1, pp. 78-95, 2001.

[10] Z. Hu, Z. Han, and Z. Tian, "Fault detection and diagnosis for singular stochastic systems via B-spline expansions," ISA Transactions, vol. 48, no. 4, pp. 519-524, 2009.

[11] M. L. Corradini and G. Orlando, "Actuator failure identification and compensation through sliding modes," IEEE Transactions on Control Systems Technology, vol. 15, no. 1, pp. 184-190, 2007.

[12] P. Mhaskara, C. McFallb, A. Ganib, P. D. Christofidesb, and J. F. Davis, "Isolation and handling of actuator faults in nonlinear systems," Automatica, vol. 44, no. 1, pp. 53-62, 2008.

[13] F. Tao and Q. Zhao, "Design of stochastic fault tolerant control for $\mathrm{H}_{2}$ performance," International Journal of Robust and Nonlinear Control, vol. 17, no. 1, pp. 1-24, 2007.

[14] Z.-H. Mao and B. Jiang, "Fault estimation and accommodation for networked control systems with transfer delay," Acta Automatica Sinica, vol. 33, no. 7, pp. 738-743, 2007.
[15] W. Cai, X. H. Liao, and Y. D. Song, "Indirect robust adaptive fault-tolerant control for attitude tracking of spacecraft," Journal of Guidance, Control, and Dynamics, vol. 31, no. 5, pp. 1456-1463, 2008.

[16] X. Su, L. Wu, and P. Shi, "Senor networks with random link failures: distributed filtering for T-S fuzzy systems," IEEE Transactions on Industrial Informatics, vol. 9, no. 3, pp. 17391750, 2013.

[17] M. Zhong, S. Li, and Y. Zhao, "Robust $H_{\infty}$ fault detection for uncertain LDTV systems using Krein space approach," International Journal of Innovative Computing, Information and Control, vol. 9, no. 4, pp. 1637-1649, 2013.

[18] D. P. Looze, J. L. Weiss, J. S. Eterno, and N. M. Barrett, "An automatic redesign approach for restructurable control systems," IEEE Control Systems Magazine, vol. 5, no. 2, pp. 1622, 1985.

[19] J. D. Boskovic, S.-H. Yu, and R. K. Mehra, "Stable adaptive faulttolerant control of over actuated aircraft using multiple models, switching and tuning," in Proceedings of the AIAA Guidance, Navigation and Control Conference, pp. 739-749, Boston, Mass, USA, 1998.

[20] J. D. Boskovic and R. K. Mehra, "Stable multiple model adaptive flight control for accommodation of a large class of control effector failures," in Proceedings of the American Control Conference (ACC ,99), pp. 1920-1924, San Diego, Calif, USA, June 1999.

[21] J. D. Boskovic, J. A. Jackson, R. K. Mehra, and N. T. Nguyen, "Multiple-model adaptive fault-tolerant control of a planetary lander," Journal of Guidance, Control, and Dynamics, vol. 32, no. 6, pp. 1812-1826, 2009.

[22] M. A. Demetriou and M. M. Polycarpou, "Incipient fault diagnosis of dynamical systems using online approximators," IEEE Transactions on Automatic Control, vol. 43, no. 11, pp. 16121617, 1998.

[23] A. T. Vemuri and M. M. Polycarpou, "Robust nonlinear fault diagnosis in input-output systems," International Journal of Control, vol. 68, no. 2, pp. 343-360, 1997.

[24] X. Zhang, T. Parisini, and M. M. Polycarpou, "Adaptive fault-tolerant control of nonlinear uncertain systems: an information-based diagnostic approach," IEEE Transactions on Automatic Control, vol. 49, no. 8, pp. 1259-1274, 2004.

[25] M. L. Corradini and G. Orlando, "A sliding mode controller for actuator failure compensation," in Proceedings of the 42nd IEEE Conference on Decision and Control (CDC '03), pp. 4291-4296, Maui, Hawaii, USA, December 2003.

[26] Q. Zhao and J. Jiang, "Reliable state feedback control system design against actuator failures," Automatica, vol. 34, no. 10, pp. 1267-1272, 1998.

[27] G. Tao, S. Chen, and S. M. Joshi, "An adaptive actuator failure compensation controller using output feedback," IEEE Transactions on Automatic Control, vol. 47, no. 3, pp. 506-511, 2002.

[28] G.-H. Yang and D. Ye, "Reliable $H_{\infty}$ control of linear systems with adaptive mechanism," IEEE Transactions on Automatic Control, vol. 55, no. 1, pp. 242-247, 2010.

[29] X. Tang, G. Tao, and S. M. Joshi, "Adaptive actuator failure compensation for parametric strict feedback systems and an aircraft application," Automatica, vol. 39, no. 11, pp. 1975-1982, 2003.

[30] X. D. Tang, G. Tao, and S. M. Joshi, "Adaptive actuator failure compensation for nonlinear MIMO systems with an aircraft 
control application," Automatica, vol. 43, no. 11, pp. 1869-1883, 2007.

[31] G. Wang and Q. Zhang, "Robust control of uncertain singular stochastic systems with Markovian switching via proportionalderivative state feedback," IET Control Theory \& Applications, vol. 6, no. 8, pp. 1089-1096, 2012.

[32] C. Wang and Y. Shen, "Delay partitioning approach to robust stability analysis for uncertain stochastic systems with interval time-varying delay," IET Control Theory \& Applications, vol. 6, no. 7, pp. 875-883, 2012.

[33] R. Sakthivel, K. Mathiyalagan, and S. M. Anthoni, "Robust $H_{\infty}$ control for uncertain discrete-time stochastic neural networks with time-varying delays," IET Control Theory \& Applications, vol. 6, no. 9, pp. 1220-1228, 2012.

[34] J. Hu, Z. Wang, H. Gao, and L. K. Stergioulas, "Robust sliding mode control for discrete stochastic systems with mixed time delays, randomly occurring uncertainties, and randomly occurring nonlinearities," IEEE Transactions on Industrial Electronics, vol. 59, no. 7, pp. 3008-3015, 2012.

[35] R. Z. Khasminskii, Stochastic Stability of Differential Equations, Sijthoff and Noordhoff, Amsterdam, The Netherlands, 1980.

[36] P. A. Ioannou and J. Sun, Robust Adaptive Control, PrenticeHall, Englewood Cliffs, NJ, USA, 1996. 


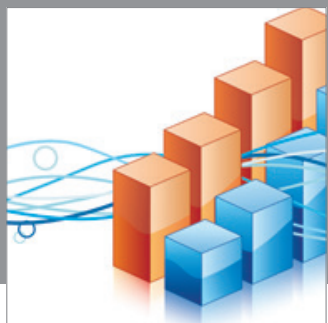

Advances in

Operations Research

mansans



The Scientific World Journal
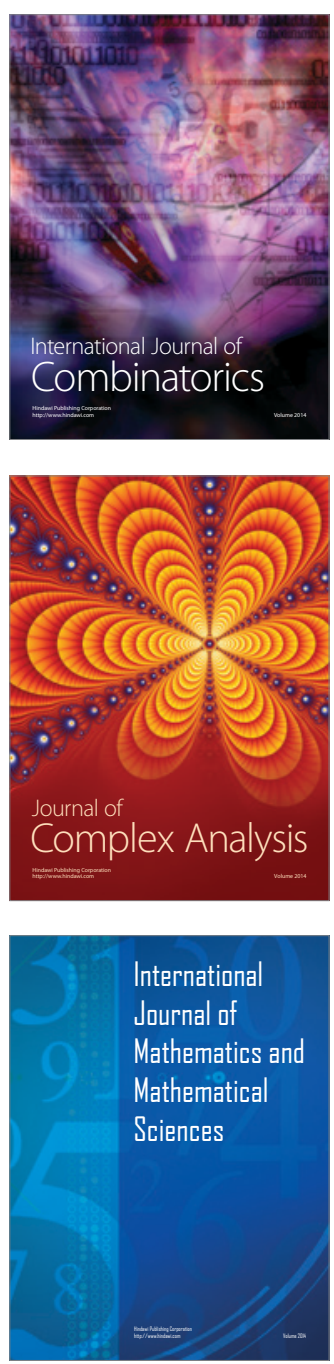
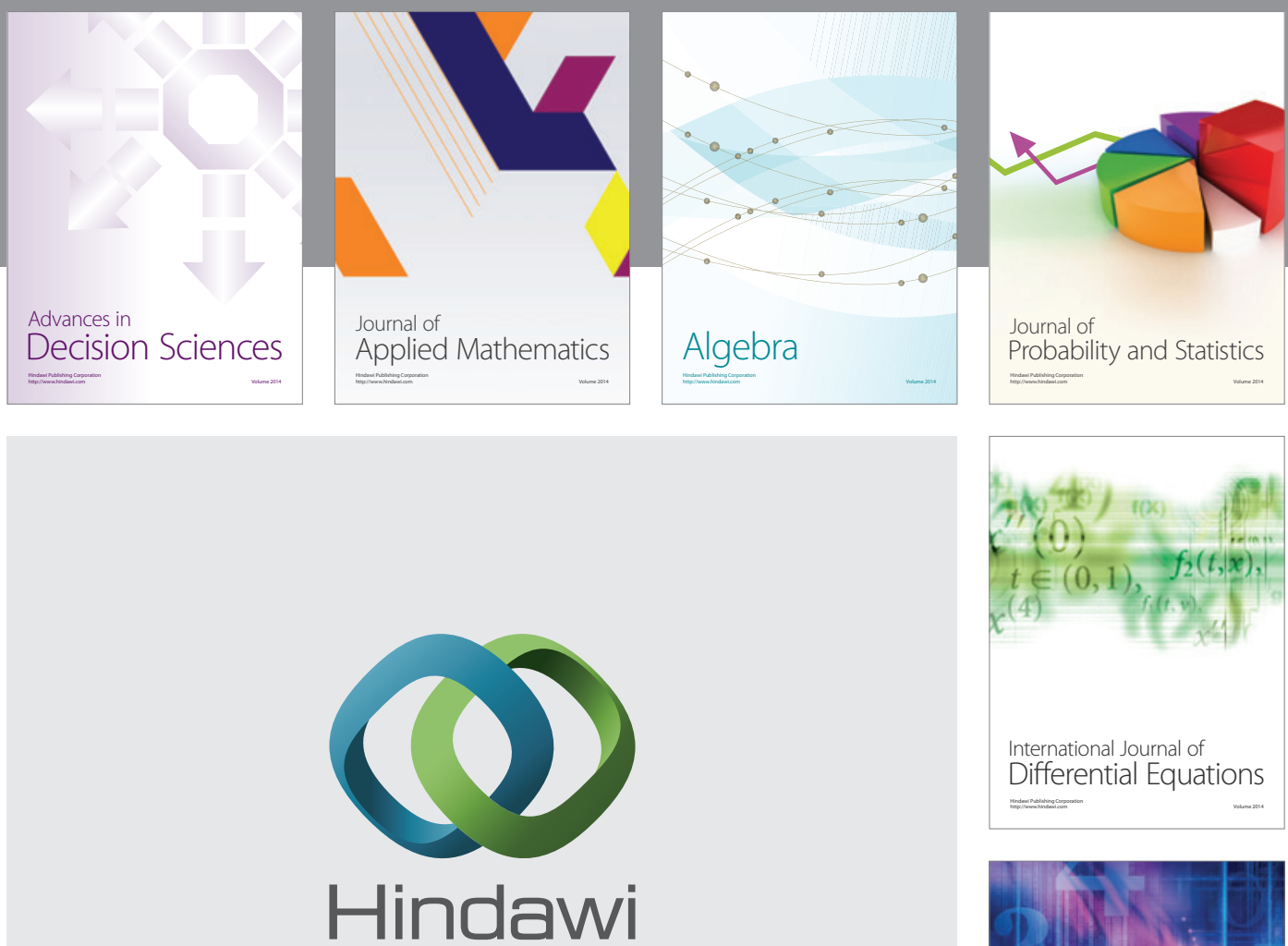

Submit your manuscripts at http://www.hindawi.com
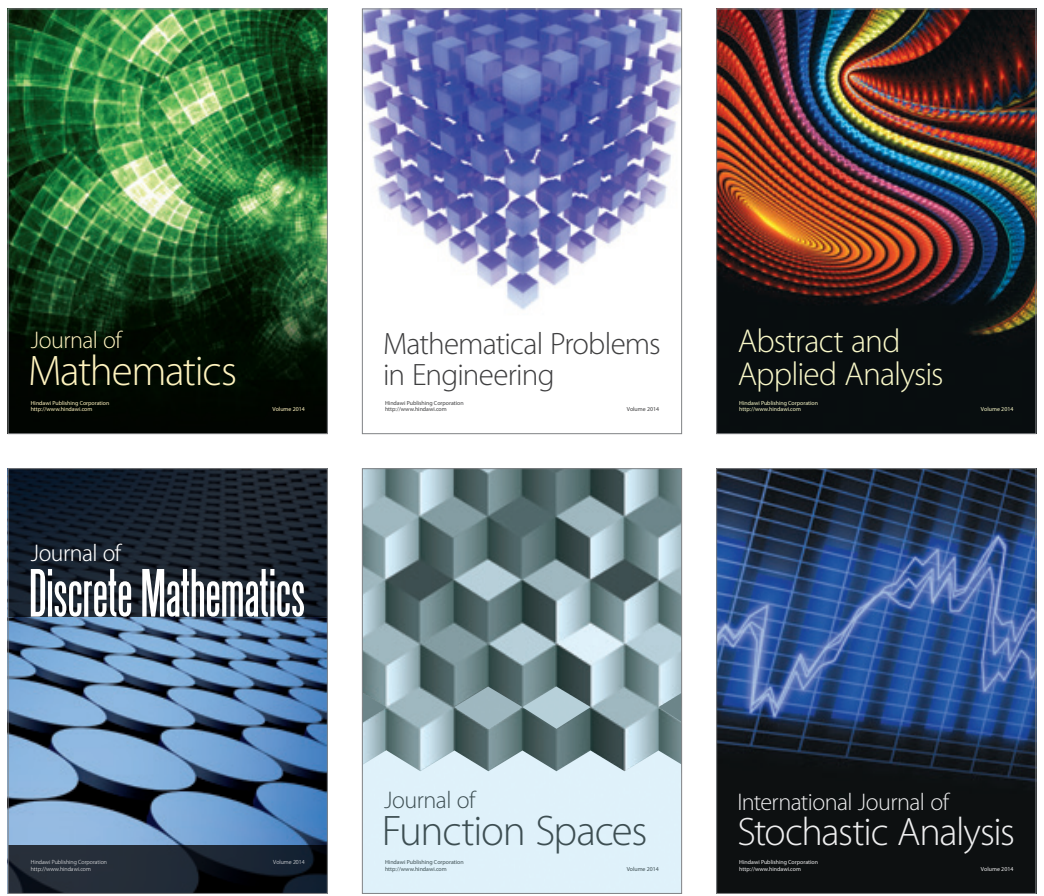

Journal of

Function Spaces

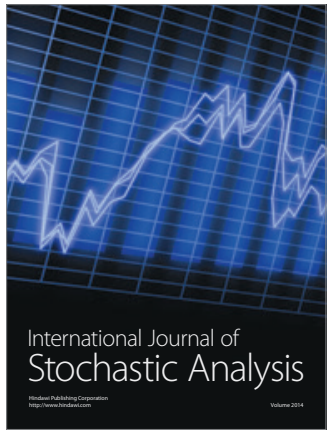

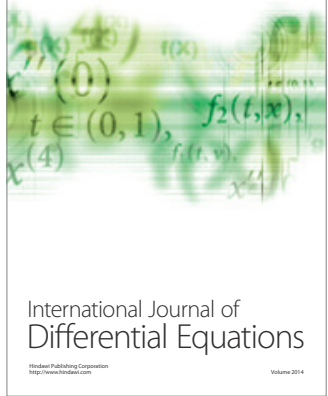
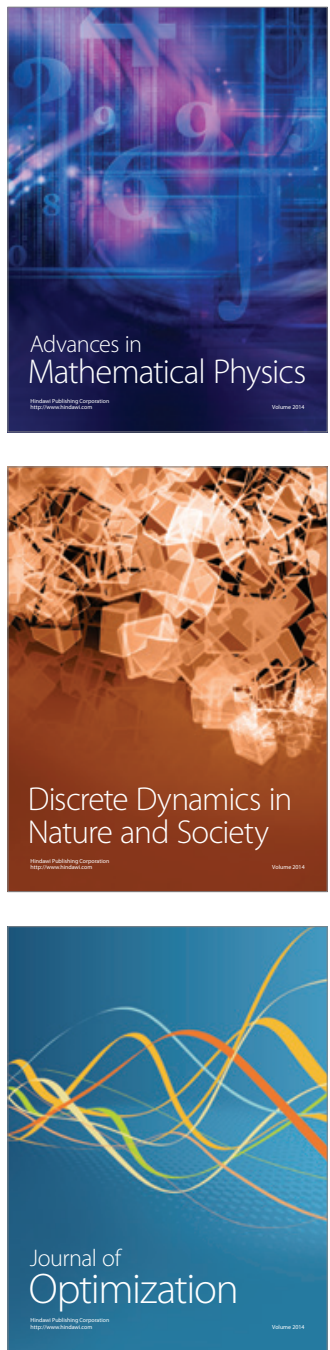